\title{
Explicitly covariant form of the integral Maxwell equations
}

\author{
J. L. Jiménez ${ }^{a}$ and G. Monsivais ${ }^{b}$ \\ ${ }^{a}$ Departamento de Física, División de Ciencias Básicas e Ingeniería, Universidad Autónoma Metropolitana, Iztapalapa, \\ Avenida San Rafael Atlixco 186, Col. Vicentina 09340 Iztapalapa CDMX. \\ e-mail: jlj@xanum.uam.mx \\ ${ }^{b}$ Instituto de Física, Universidad Nacional Autónoma de México, \\ Apartado Postal 20-364, 01000 CDMX, \\ e-mail: monsi@fisica.unam.mx
}

Received 4 April 2020; accepted 18 August 2020

\begin{abstract}
We analyze in detail the covariance of the integral forms of the Maxwell Equations. Different forms of writing the integral Maxwell equations in explicitly covariant form are analyzed. First, we show how one of these ways can be obtained from the differential Maxwell equations, both from their usual three vector form as from their covariant four vector form. Then we discuss how this covariant integral Maxwell equations can be obtained from usual integral Maxwell equations. We emphasize the necessity to write the usual integral equations without time derivatives. The integrations regions in the three-dimensional space and time are identified with parts of the same hyper-surface in four-dimensional space-time. This point is carefully analyzed. Later we discuss other forms of the integral Maxwell equations. We show how these new versions can be expressed in an explicitly covariant form.
\end{abstract}

Keywords: Maxwell integral equations in explicitly covariant form; covariant Maxwell integral equations; classical integral electrodynamics in covariant form.

PACS: 03.30+p; 03.50.De; 41.20

DOI: https://doi.org/10.31349/RevMexFisE.18.76

\section{Introduction}

It is a historical fact that relativity theory emerged from classical electrodynamics. Thus, an inertial frame, in relativity theory, is one relative to which Maxwell equations hold, and we change from one inertial frame to another by means of Lorentz transformations [1]. After the introduction by Einstein of space-time, classical electrodynamics could be written in the language of tensor analysis in space-time. Due to the intrinsic property of the tensors, this form of writing the equations has the advantage that one can be sure that the equations have the same form in all inertial frames. For this reason, we call this formulation explicitly covariant. In contrast, writing the Maxwell Equations in the usual three-dimensional form does not explicitly exhibit the covariance. Unfortunately, in most texts on electromagnetism [210] or relativity [11-14] we find only the covariant formulation of the differential Maxwell equations. As far as we know, only Aharoni [15] and Ley [16] have discussed the integral Maxwell equations in their covariant form. Nevertheless, those studies are incomplete. In Ref. [15] only the case where no sources are present is discussed. Furthermore, its formulation cannot be directly used to obtain the correct form for the case where sources are present due to an error in one of its expressions as discussed in sub-section 2.2. In Ref. [16], the expressions obtained are not properly integral forms, since such expressions also contain derivatives, i.e., they are integro-differential equations. Furthermore, in none of these references is it described the role of the surfaces and hyper-surfaces embedded in the four-dimensional space-time over which the integrals are performed, a crucial point in this formulation. Neither is discussed the relation between the covariant and non-covariant formulation, particularly about the integration regions involved in both formulations.

One reason for which the covariance of the integral forms are less studied is that the differential equations refer to points of space and time that can be trivially written covariantly, while the integral equations refer to continuous sets of points in the space and time. How these equations can be expressed covariantly is not as evident as in the differential formulation. Nevertheless, as we will see, the transition can be done without many difficulties if it is recognized how the different integration regions that appear in the conventional formulation can be chosen such that all of them corresponds to parts of a single hyper-surface embedded in the four-dimensional space-time. The objective of this paper is to discuss all these points in detail.

Our presentation is as follows. Section 2 is focused to the derivation of the covariant integral forms. Nevertheless, as a preamble and in order to introduce our notation, in Subsec. 2.1, we first present a brief review of the procedure to derive the explicitly covariant differential Maxwell equations (ECDME) starting from the usual three-dimensional differential Maxwell equations (DME3). In Subsec. 2.2 we derive the explicitly covariant integral Maxwell equations (ECIME) starting from the ECDME. This formulation is a generalization of the one of Aharoni [15] and goes beyond the discussion of Ley [16]. The objective of Subsec. 2.3 is to analyze the formulation of the previous sub-section from the point of view of the usual three-dimensional differential equations. In 
Subsec. 2.4 we derive the ECIME starting from the usual three-dimensional integral Maxwell equations IME3. Subsection 2.4 is the most important and interesting. It contains many crucial and non-trivial details that must be considered. Unfortunately, their discussion is almost absent in the literature. The summary of Sec. 2 can be written symbolically as

2.1 Differential three-vector $\rightarrow$ Differential four-vector $(\mathrm{DME} 3 \rightarrow$ ECDME)

2.2 Differential four-vector $\rightarrow$ Integral four-vector $(\mathrm{ECDME} \rightarrow \mathrm{ECIME})$

2.3 Differential three-vector $\rightarrow$ Integral four-vector (DME3 $\rightarrow$ ECIME)

2.4 Integral three-vector $\rightarrow$ Integral four-vector (IME3 $\rightarrow$ ECIME)

In Subsec. 3.1 we obtain other forms of the non-covariant integral Maxwell equations, which are not usually discussed in the literature. In Subsec. 3.2 we obtain a new form of explicitly covariant integral Maxwell equations starting from the usual three-dimensional differential equations. Finally, in Subsec. 3.3 we obtain this new covariant expression from the covariant differential equations. With this we offer a different perspective that we hope will leads to understand the close relationship between electromagnetism in its integral form and special relativity.

\section{Electromagnetism in explicitly covariant in- tegral form}

\subsection{Preamble. Explicitly covariant differential Maxwell equations from the usual differential Maxwell equa- tions}

In order to make the subsequent analysis clearer and to introduce our notation, we recall the procedure to exhibit the covariance of the Maxwell equations in differential form starting from the usual three-dimensional differential form. In this work the Latin indexes (lower or upper) take values on the set $\{1,2,3\}$ while the Greek indexes take values on the set $\{0,1,2,3\}$. Furthermore, we follow the Einstein summation convention which implies summation over repeated covariant and contravariant indexes. It is important to be aware that expressing the Maxwell equations by means of fourdimensional mathematical objects does not entail apriori a relativistic generalization neither the required properties of covariance [17]. For this it is necessary that the objects were tensors with respect to the Lorentz transformations. Only in this way one guarantees that the equations are covariant. In general, an arbitrary four-component object is not a tensor. To know if the object is a tensor it is necessary to prove that the law of transformation of the tensors is satisfied. In what follows, whenever an object which is a tensor is used, such characteristic will be explicitly stated without proving that it satisfies the required transformation law.

We proceed as follows. We identify first the Cartesian components of the following four components objects, which are contravariant tensors of first rank (we use Heaviside units with $c=1$ ).

$$
\begin{aligned}
& \left(x^{\mu}\right)=\left(x^{0}, x^{1}, x^{2}, x^{3}\right)=(t, x, y, z)=(t, \mathbf{r}) \\
& \text { four-vector of coordinates } \\
& \left(j^{\mu}\right)=\left(j^{0}, j^{1}, j^{2}, j^{3}\right)=\left(\rho, j_{x}, j_{y}, j_{z}\right)=(\rho, \mathbf{j}) \\
& \text { density current four-vector }
\end{aligned}
$$

and the covariant tensor of first rank

$$
\begin{aligned}
\left(\partial_{\mu}\right) & =\left(\partial_{0}, \partial_{1}, \partial_{2}, \partial_{3}\right)=\left(\frac{\partial}{\partial t}, \frac{\partial}{\partial x}, \frac{\partial}{\partial y}, \frac{\partial}{\partial z}\right) \\
& =\left(\frac{\partial}{\partial t}, \nabla\right) \text { four-nabla operator. }
\end{aligned}
$$

We also need the contravariant second-rank antisymmetric electromagnetic field tensor $F^{\mu v}$. It has the matrix elements

$$
\begin{aligned}
& F^{m 0}=E^{m}=E_{m}, \\
& F^{m n}=-\grave{o}^{m n \ell} B_{\ell},
\end{aligned}
$$

where $E_{m}$ and $B_{m}$ are the Cartesian components of the electric and the magnetic induction fields $\mathbf{E}$ and $\mathbf{B}$ respectively. The symbol o ${ }^{m n \ell}$ denotes the totally antisymmetric third rank tensor

$$
\grave{\mathrm{o}}^{m n \ell}=\left\{\begin{array}{ll}
1 & \text { for } m=1, n=2, \ell=3 \text { or any } \\
-1 & \text { for any odd permutation } \\
0 & \text { if any two indices are equal }
\end{array} .\right.
$$

In matrix form the tensor $F^{\mu v}$ is writing as

$$
\left(F^{\mu \nu}\right)=\left(\begin{array}{cccc}
0 & -E_{x} & -E_{y} & -E_{z} \\
E_{x} & 0 & -B_{z} & B_{y} \\
E_{y} & B_{z} & 0 & -B_{x} \\
E_{z} & -B_{y} & B_{x} & 0
\end{array}\right) .
$$

The totally antisymmetric fourth-rank tensor $\grave{o}^{\mu \nu \lambda \delta}$ is also useful; it is defined as an obvious generalization of o ${ }^{m n \ell}$ Furthermore, one can get the corresponding covariant tensor of a given contravariant tensor by using the metric tensor $g_{\alpha \beta}$. This tensor can be defined by means of a diagonal matrix whose diagonal elements are $g_{00}=1$ and $g_{11}=g_{22}=$ $g_{33}=-1$. For example, the electromagnetic tensor with two covariant indices $F_{\gamma \delta}$ is equal to $F_{\gamma \delta}=g_{\gamma \mu} g_{\delta \nu} F^{\mu \nu}$ and it is easily shown that his elements can be obtained from $F^{\mu v}$ by putting $\mathbf{E} \rightarrow-\mathbf{E}$. Similarly, the totally antisymmetric fourth-rank tensor with covariant indices $\grave{\mathrm{o}}_{\mu \nu \lambda \delta}$ is equal to $\grave{\mathrm{o}}_{\alpha \beta \gamma \delta}=g_{\alpha k} g_{\beta \lambda} g_{\gamma \mu} g_{\delta \nu} \grave{\mathrm{o}}^{k \lambda \mu \nu}$ and is easily shown that $\grave{\mathrm{o}}_{\alpha \beta \gamma \delta}=-\grave{\mathrm{o}}^{\alpha \beta \gamma \delta}$. 
With these elements, the Cartesian components of the inhomogeneous Maxwell equations:

$$
\begin{aligned}
\nabla \cdot \mathbf{E} & =\rho, \\
(\nabla \times \mathbf{B})_{k} & =\frac{\partial E_{k}}{\partial t+j_{k}},
\end{aligned}
$$

can be written as $\partial_{\mu} F^{\mu 0}=J^{0}$ and $\partial_{\mu} F^{\mu k}=J^{k}$ respectively. These four equations can be written as a single expression

$$
\partial_{\mu} F^{\mu v}=J^{\nu}
$$

which are the desired four explicitly covariant inhomogeneous differential Maxwell equations.

The homogeneous equations

$$
\begin{aligned}
\nabla \cdot \mathbf{B} & =0 \\
(\nabla \times \mathbf{E})_{k} & =-\frac{\partial B_{k}}{\partial t},
\end{aligned}
$$

can be written in covariant form using the dual electromagnetic field tensor $\mathfrak{F}^{\mu \nu}$ whose elements are again the components of $\mathbf{E}$ and $\mathbf{B}$ but arranged differently. This tensor is easily constructed using the tensors $F_{\gamma \delta}$ and $\mathrm{ò}_{\mu \nu \lambda \delta}$. The relation is

$$
\mathfrak{F}^{\mu \nu}=\frac{1}{2} \grave{\mathrm{o}}^{\mu \nu \gamma \delta} F_{\gamma \delta}
$$

which implies the correspondences

$$
\begin{aligned}
\mathfrak{F}^{m 0} & =B^{m}=B_{m}, \\
\mathfrak{F}^{m n} & =\grave{\mathrm{o}}^{m n \ell} E_{\ell} .
\end{aligned}
$$

In matrix form we have

$$
\left(\mathfrak{F}^{\mu \nu}\right)=\left(\begin{array}{cccc}
0 & -B_{x} & -B_{y} & -B_{z} \\
B_{x} & 0 & E_{z} & -E_{y} \\
B_{y} & -E_{z} & 0 & E_{x} \\
B_{z} & E_{y} & -E_{x} & 0
\end{array}\right)
$$

Then, the four homogeneous Maxwell Eqs. (11) and (12) can be written in covariant form as

$$
\partial_{\mu} \mathfrak{F}^{\mu \nu}=0
$$

\subsection{Explicitly covariant integral Maxwell equations from explicitly covariant differential Maxwell equa- tions}

In this sub-section we derive the ECIME starting from the ECDME: Eqs. (10) and (17). This formulation is a generalization of the one of Aharoni [15] since we include the case in which the sources are different from zero. We also go beyond the discussion of Ley [16] since we obtain properly integral forms and not only integro-differential equations as is done in [16]. Additionally, we describe in detail the surfaces and hyper-surfaces embedded in the four-dimensional space-time over which the integrals are done.
Equations (10) and (17) can be integrated in the fourdimensional space-time over different regions. For example they can be integrated over open or closed lines, open or closed surfaces, open or closed hyper-surfaces or hypervolumes. However, usually only certain integration regions are considered. This is so because they are the ones that are directly related with the experimental procedures (see Subsec. 2.4) or because one is interested in considering regions in which the role of the spatial coordinates and the time coordinate is symmetrical (as will be discussed in this and in the next sub-sections).

In the following we will integrate Eq. (10) over an arbitrary hyper-surface by means of a three-dimensional hypersurface $\mathbf{H}_{s} \subset \mathbf{R}^{4}$ integral. See definition of this kind of integral in Appendix A. We assume that this hyper-surface is the image of a three-dimensional region $V_{u v w} \subset \mathbf{R}^{3}$ by means of the mapping

$$
\overrightarrow{\mathbf{x}}=\overrightarrow{\mathbf{X}}(\mathbf{u})=\left(X^{0}(\mathbf{u}), X^{1}(\mathbf{u}), X^{2}(\mathbf{u}), X^{3}(\mathbf{u})\right),
$$

where $\overrightarrow{\mathbf{x}}=\left(x^{\mu}\right)=\left(x^{0}, x^{1}, x^{2}, x^{3}\right)$ is the four-vector defined in Eq. (1), $\overrightarrow{\mathbf{X}}: V_{u v w} \rightarrow \mathbf{H}_{S}$ is a four-component function depending on the three-dimensional variable $\mathbf{u}=(u, v, w) \in$ $V_{\mathbf{u}}$ being $u, v, w$ the three parameters generating $\mathbf{H}_{S}$. As mentioned, $\mathbf{H}_{S}$ is totally arbitrary, the only restriction that is asked is that it can be parameterized as indicated above. We denote as $\partial V_{u v w}$, the boundary of $V_{u v w}$. We also assume that not all the points of the set $\overrightarrow{\mathbf{X}}\left(\partial V_{u v w}\right)$ are double points (possibly none of them) and therefore $\mathbf{H}_{S}$ is an open region. The surface surrounding $\mathbf{H}_{S}$ denoted as $\partial \mathbf{H}_{S}$ is a closed surface which is generated by the subset of $\overrightarrow{\mathbf{X}}\left(\partial V_{u v w}\right)$ formed of all the non-double points. Since three-dimensional hypersurface integrals are to be used, the integrations elements must be differential of measure of hyper-surface and, in order to have covariant expressions, they must be the components of a tensor. The candidate is the antisymmetric contravariant third-rank tensor $\left(d V^{\alpha \beta \gamma}\right)$ whose elements are defined by means of the determinant

$$
d V^{\alpha \beta \gamma}=\left(\varepsilon^{i j k} \frac{\partial X^{\alpha}}{\partial u^{i}} \frac{\partial X^{\beta}}{\partial u^{j}} \frac{\partial X^{\gamma}}{\partial u^{k}}\right) d u^{1} d u^{2} d u^{3}
$$

where $u^{1}=u, u^{2}=v, u^{3}=w$. This tensor has only four independent components different from zero. The sign of $d V^{\alpha \beta \gamma}$ is not an intrinsic property since it depends of the order assigned to the parameters $u^{1}, u^{2}, u^{3}$. Instead of that, its magnitude $\left|d V^{\alpha \beta \gamma}\right|$ is equal to the measure of the projection of the differential of hyper-surface on the hyper-plane $\alpha \beta \gamma$, For our purposes, however, it is more useful to construct a covariant tensor and to have its indices assigned in another way. This is easily accomplished by means of the dual covariant one-rank tensor $d \Psi_{\delta}$ defined as $d \Psi_{\delta}=(1 / 3 !) \grave{o ̀}_{\alpha \beta \gamma \delta} d V^{\alpha \beta \gamma}$. 
This implies

$$
\begin{aligned}
& d \Downarrow_{0}=d V^{123} \\
& d \Downarrow_{1}=-d V^{230} \\
& d \nabla_{2}=d V^{301} \\
& d \nabla_{3}=-d V^{012}
\end{aligned}
$$

The explicit form of these differential elements depends of the parameterization to be used. For example, when the parameterization is $(u, v, w)=(x, y, z)$ one obtains $d \mathbb{\Psi}_{0}=$ $d x d y d z$ and $d \mathbb{\Psi}_{3}=-\partial X^{0} / \partial z d x d y d z$ while when the parameterization is $(u, v, w)=(t, x, y)$ one obtains $d \mathbb{t}_{3}=$ $d t d x d y$, etc.

We now multiply Eq. (10) by $d \mathbb{W}_{\nu}$ and we sum over the index $\nu$. After integration over the hyper-surface $\mathbf{H}_{S}$ we obtain

$$
\iiint_{\mathbf{H}_{S}} \partial_{\mu} F^{\mu \nu} d \mathbb{\Psi}_{\nu}=\iiint_{\mathbf{H}_{S}} j^{\mu} d \mathbb{\Psi}_{\nu}
$$

Next we will use the following integral theorem that relates a hyper-surface integral over an open three-dimensional hyper-surface $\mathbf{H}_{S} \subset \mathbf{R}^{4}$ with a surface integral over the twodimensional surface $\partial \mathbf{H}_{S} \subset \mathbf{R}^{4}$ surrounding $\mathbf{H}_{S}$,

$$
2 \iiint_{\mathbf{H}_{S}} \partial_{\mu} \Phi^{\mu \nu} d \mathbb{t}_{\nu}=\iint_{\partial \mathbf{H}_{S}} \Phi^{\mu \nu} d \sigma_{\mu \nu},
$$

being $\Phi^{\mu \nu}$ an arbitrary antisymmetric function. This theorem is written with an error in Ref. [15], it lacks the factor of 2. The tensor $d \theta_{\mu \nu}$ is the dual of the contravariant antisymmetric two-rank tensor $d \sigma^{\mu \nu}$ defined by means of the determinant

$$
d \sigma^{\mu \nu}=\left(\varepsilon^{i j} \frac{\partial \Xi^{\mu}}{\partial \theta^{i}} \frac{\partial \Xi^{\nu}}{\partial \theta^{j}}\right) d \theta^{1} d \theta^{2}
$$

where $\vec{\Xi}:\left\{\left(\theta^{1}, \theta^{2}\right)\right\} \rightarrow \partial \mathbf{H}_{S} \subset \mathbf{R}^{4}$ is the mapping generating $\partial \mathbf{H}_{S}$ and $\theta^{1}, \theta^{2}$ are the two parameters. This tensor has only six independent components different form zero. The magnitude $\left|d \theta_{\mu \nu}\right|$ is the differential of area of the projection of $\partial \mathbf{H}_{S}$ on the plane $\mu \nu$ The dual tensor $d \theta_{\mu \nu}$ is defined as $d \theta_{\mu \nu}=(1 / 2) \grave{\mathrm{o}}_{\mu \nu \alpha \beta} d \sigma^{\alpha \beta}$ which implies

$$
\begin{aligned}
& d \sigma_{01}=-d \sigma^{23}, \quad d \sigma_{12}=-d \sigma^{03}, \\
& d \sigma_{02}=d \sigma^{13}, \quad d \sigma_{13}=d \sigma^{02}, \\
& d \theta_{03}=-d \sigma^{12}, \quad d \sigma_{23}=-d \sigma^{01} .
\end{aligned}
$$

From Eqs. (20), (21) we finally obtain the inhomogeneous ECIME

$$
\iint_{\partial \mathbf{H}_{S}} F^{\mu \nu} d \nabla_{\mu \nu}=2 \iiint_{\mathbf{H}_{S}} j^{\nu} d \nabla_{\nu},
$$

where $\mathbf{H}_{S}$ is an arbitrary hyper-surface. It is worth mentioning that for some hyper-surfaces the RHS of Eq. (26) can be equal to zero even though the sources are different from zero (see appendix B). In this case, Eq. (26) is not a proper Maxwell integral equation and it is necessary to search for them as is done in Sec. 3. Nevertheless, for the case in which the sources are equal to zero, Eq. (26) reduces to the Aharoni result and the error in the factor 2 disappears.

Following the same procedure, we obtain for the homogeneous

$$
\int_{\partial \mathbf{H}_{S}} \mathfrak{F}^{\mu \nu} d \theta_{\mu \nu}=0 .
$$

The covariance of expressions (26) and (27) is a consequence of the transform properties of the tensors $F^{\mu \nu}, d \theta_{\mu \nu}$, etc.

\subsection{Explicitly covariant integral Maxwell equations from the usual differential Maxwell equations}

The objective of this sub-section is to discuss the derivation of the ECIME: Eqs. (26) and (27) from the point of view of the usual DME3 and to analyze the relationship between this formulation and the one discussed in the previous subsection. The integration regions involved in both treatments is also described in detail. This and the next sub-sections contain several fundamental and subtle points.

We will start from the usual three-dimensional form of the inhomogeneous Maxwell equations (8) and (9). As we want to compare with the expressions derived in the previous sub-section, what must be done is to integrate on the same region in both formulations. So, we will integrate the threedimensional Eqs. (8) and (9) over the hyper-surface $\mathbf{H}_{S}$ of Subsec. 2.2. Multiplying Eqs. (8) and (9) respectively by the elements $d \mathbb{W}_{0}$ and $d \mathbb{W}_{k}$ defined in Eq. (19) and summing over $k$, we obtain

$$
\begin{gathered}
\nabla \cdot \mathbf{E} d \mathbb{\nabla}_{0}=\rho d \mathbb{\nabla}_{0}=j^{0} d \mathbb{\nabla}_{0}, \\
(\nabla \times B)_{k} d \mathbb{\nabla}_{k}-\frac{\partial E_{k}}{\partial t} d \nabla_{k}=j_{x} d \mathbb{\nabla}_{k},
\end{gathered}
$$

where $k=1,2,3$. The sum of these four equations gives

$$
(\nabla \times \mathbf{B})_{k} d \mathbb{\nabla}_{k}-\frac{\partial E_{k}}{\partial t} d \nabla_{k}+\nabla \cdot \mathbf{E} d \mathbb{V}_{0}=j^{\nu} d \mathbb{\forall}_{\nu}
$$

Now we integrate over the hyper-surface $\mathbf{H}_{S}$ In the integral of the function $(\nabla \times \mathbf{B})_{1} d \mathbb{W}_{1}$ we use the parameters $\left(u^{1}, u^{2}, u^{3}\right)=(t, y, z)$ and therefore

$$
d \nabla_{1}=d y d z d t=\cos \alpha d S d t=n_{1} d S d t,
$$

being $\alpha$ the angle between the differential surface $d S$ and its projection on the plane $Y Z$. The symbol $n_{1}$ is the first component of the unitary vector $\hat{\mathbf{n}}=(\cos \alpha, \cos \beta, \cos \gamma)$ which is perpendicular to $d S$. So,

$$
\iiint_{\mathbf{H}_{S}}(\nabla \times \mathbf{B})_{1} d \mathbb{\Psi}_{1}=\iiint_{\mathbf{H}_{S}}(\nabla \times \mathbf{B})_{1} n_{1} d S d t .
$$


The other terms can be handled in a similar form. For example, in the integral of the function $(\nabla \times \mathbf{B})_{2} d \mathbb{t}_{2}$ we use the parameters $\left(u^{1}, u^{2}, u^{3}\right)=(t, y, z)$ and in the integral of the function $\nabla \cdot \mathbf{E} d \mathbb{\mathbb { t }}_{0}$ we use the parameters $\left(u^{1}, u^{2}, u^{3}\right)=$ $(x, y, z)$. This leads to

$$
\begin{gathered}
\iiint_{\mathbf{H}_{S}}(\nabla \times \mathbf{B}) \cdot \hat{\mathbf{n}} d S d t-\iiint_{\mathbf{H}_{S}} \frac{\partial \mathbf{E}}{\partial t} \cdot \hat{\mathbf{n}} d S d t \\
+\iiint_{\mathbf{H}_{S}} \nabla \cdot \mathbf{E} d V=\iiint_{\mathbf{H}_{S}} j^{\nu} d \mathbf{H}_{\nu} .
\end{gathered}
$$

Note that when working with the usual three-dimensional equations it is customary to add only the three Eqs. (29) and to integrate them over the open surface, while Eq. (28) is worked out separately and integrated over the volume $V$. However, since what is desired here is to compare with the results of the previous sub-section what must be done is to add up the four equations and to integrate over the same region. The RHS of Eq. (33) already has the desired form, whereas the LHS is an integro-differential expression. To convert Eq. (33) into a purely integral expression the following integral theorem will be used

$$
\iiint_{\mathbf{H}_{S}} \frac{\partial \phi}{\partial x^{\mu}} d \nabla_{\nu}-\iiint_{\mathbf{H}_{S}} \frac{\partial \phi}{\partial x^{\nu}} d \forall_{\mu}=\iint_{\partial \mathbf{H}_{S}} \phi d \nabla_{\mu \nu} .
$$

This theorem is one of the generalizations of the usual divergence theorem in $\mathbf{R}^{3}$ which can be written for example as

$$
\iiint_{V} \frac{\partial \phi}{\partial x} d V=\iiint_{\partial V} \phi d y d z
$$

A more detailed discussion on this point is included in this paper as quotes (Refs. [18,19]). Note that Eq. (34) has an extra term respect to this usual theorem because the integral is over the hyper-surface $\mathbf{H}_{S} \subset \mathbf{R}^{4}$ instead of the volume $V \subset \mathbf{R}^{3}$. It is also worth noting that theorem (34) can be used to demonstrate expression (21) (which is the one incorrectly written in Ref. [15]); it is enough to apply it to an anti-symmetric function of two indices.

Applying theorem (34) to the first integral of (33), after some cumbersome algebra, we obtain

$$
\begin{aligned}
\iiint_{\mathbf{H}_{S}}(\nabla \times \mathbf{B}) \cdot \hat{\mathbf{n}} d S d t & =-\iiint_{\mathbf{H}_{S}}(\nabla \times \mathbf{B}) \cdot \hat{\mathbf{n}} d S d t \\
& -2 \iint_{\partial \mathbf{H}_{S}} \mathbf{B} \cdot d \mathbf{I} d t, \\
\iiint_{\mathbf{H}_{S}}(\nabla \times \mathbf{B}) \cdot \hat{\mathbf{n}} d S d t & =-\int_{\partial \mathbf{H}_{S}} \mathbf{B} \cdot d \mathbf{I} d t .
\end{aligned}
$$

We now apply (34) to the second integral of (33), we ob- tain

$$
\begin{aligned}
-\iiint_{\mathbf{H}_{S}} \frac{\partial \mathbf{E}}{\partial t} \cdot \hat{\mathbf{n}} d S d t & =\iint_{\partial \mathbf{H}_{S}} \mathbf{E} \cdot \hat{\mathbf{n}} d S \\
& -\iiint_{\mathbf{H}_{S}}(\nabla \cdot \mathbf{E}) d V .
\end{aligned}
$$

Note that this result clearly indicates that

$$
\iint_{\partial \mathbf{H}_{S}} \mathbf{E} \cdot \hat{\mathbf{n}} d S \neq \iiint_{\mathbf{H}_{S}}(\nabla \cdot \mathbf{E}) d V,
$$

which contrasts with the usual expression of the divergence theorem in $\mathbf{R}^{3}$. Substituting (36) and (37) into (33), obtains

$$
-\int_{\partial \mathbf{H}_{S}} \mathbf{B} \cdot d \mathbf{I} d t+\iint_{\partial \mathbf{H}_{S}} \mathbf{E} \cdot \hat{\mathbf{n}} d S=\iiint_{\mathbf{H}_{S}} j^{\nu} d \mathbb{\nabla}_{\nu}
$$

which is already a purely integral expression, and it is easy to see that the LHS can be written as

$$
\frac{1}{2} \int_{\partial \mathbf{H}_{S}} F^{\mu \nu} d \sigma_{\mu \nu}
$$

and hence Eq. (38) reduces to the inhomogeneous ECIME: Eq. (26), as desired.

Following a similar procedure we obtain that the hypersurface integral of the sum of the four Eqs. (11) and (12) multiplied respectively by the integration elements $d \mathbb{t}_{0}$ y $d \mathbb{t}_{k}$ is equal to

$$
\iiint_{\mathbf{H}_{S}}(\nabla \times \mathbf{E}) \cdot \hat{\mathbf{n}} d S d t+\iiint_{\mathbf{H}_{S}} \frac{\partial \mathbf{B}}{\partial t} \cdot \hat{\mathbf{n}} d S d t=0 .
$$

Applying theorem (34) to the first and second integrals of Eq. (39) gives respectively

$$
\begin{aligned}
\iiint_{\mathbf{H}_{S}}(\nabla \times \mathbf{E}) \cdot \hat{\mathbf{n}} d S d t & =-\iint_{\partial \mathbf{H}_{S}} \mathbf{E} \cdot d \mathbf{I} d t, \\
\iiint_{\mathbf{H}_{S}} \frac{\partial \mathbf{B}}{\partial t} \cdot \hat{\mathbf{n}} d S d t & =-\iint_{\partial \mathbf{H}_{S}} \mathbf{B} \cdot \hat{\mathbf{n}} d S .
\end{aligned}
$$

We again observe the contrast with the usual expression of the divergence theorem in $\mathbf{R}^{3}$ applied to the field $\mathbf{B}$ :

$$
\iint_{\partial V} \mathbf{B} \cdot \hat{\mathbf{n}} d S=0 .
$$

Replacing Eqs. (40) and (41) in (39) one obtains

$$
-\iint_{\partial \mathbf{H}_{S}} \mathbf{E} \cdot d \mathbf{I} d t-\iint_{\partial \mathbf{H}_{S}} \mathbf{B} \cdot \tilde{\mathbf{n}} d S=0,
$$

and it is easy to verify that using Eq. (16) this equation can be written as

$$
\frac{1}{2} \int_{\partial \mathbf{H}_{S}} \mathfrak{F}^{\mu \nu} d \nabla_{\mu \nu}=0
$$

which demonstrates Eq. (27) as desired. 


\subsection{From the non-covariant integral Maxwell equations to an explicitly covariant integral form}

While the transition from the DME3 to the ECDME, as discussed in Subsec. 2.1, is almost direct, we must proceed more carefully to obtain the ECIME starting from the usual IME3:

$$
\begin{gathered}
\int_{\partial V} \mathbf{E} \square \hat{\mathbf{n}} d S=Q \iiint_{V} \rho d V, \\
\int_{\partial S} \mathbf{B} \square d \mathbf{I}-\frac{d}{d t} \iint_{S} \mathbf{E} \square \hat{\mathbf{n}} d S=\iint_{S} \mathbf{j} \square \mathbf{n} d S, \\
\underset{\partial V}{\iint_{V} \mathbf{B} \mathbf{n} d S=0,} \\
\int_{\partial S} \mathbf{E} \square d \mathbf{I}+\frac{d}{d t} \iint_{S} \mathbf{B} \square \hat{\mathbf{n}} d S=0,
\end{gathered}
$$

where we have assumed that the surface $S \subset \mathbf{R}^{3}$ is at rest in the frame of the observer. We are using $\partial V$ for the closed surface which is the boundary of the volume $V \subset \mathbf{R}^{3}$ and $\partial S$ for the closed curve surrounding the boundary of the open surface $S$. As it is well known, the form of Eqs. (44)-(47) are closely related to the experimental observations of the electromagnetic phenomena. We must notice the following points:

a) Equations (44)-(47) involve line integrals, surface integrals and/or volume integrals.

b) Equations (45) and (47) also involve time derivatives.

c) There are two types of surface integrals: in Eqs. (44) and (46) $\partial V$ is a closed surface while in Eqs. (45) and (47) $S$ is an open surface.

The point b) is particularly important. It shows that the usual integral Maxwell equations are indeed integrodifferential equations, since time derivatives appear together with flux integrals. This fact constitutes the first obstacle to a direct identification of these equations with the explicitly covariant integral equations in space-time. Our procedure to obtain this identification is first to integrate Eqs. (45) and (47) with respect to time. This yield

$$
\int_{T} \int_{\partial S} \mathbf{B} \square d \mathbf{I} d t-\iint_{S} \mathbf{E} \square \hat{\mathbf{n}} d S=\iint_{T} \int_{S} \mathbf{j} \square \mathbf{n} d S d t
$$

and

$$
\int_{T} \int_{\partial S} \mathbf{E} \square d \mathbf{I} d t+\iint \mathbf{B} \square \hat{\mathbf{n}} d S=0 .
$$

With these integrations, in addition to avoiding time derivatives, the left side of the equations involves only integrals over two-dimensional regions. Then, Eqs. (44), (48),
(46) and (49) written in their Cartesian components become, respectively,

$$
\begin{aligned}
& \iint_{\partial V}\left(E_{x} d y d z+E_{y} d z d x+E_{z} d x d y\right) \\
&=\iiint_{V} \rho d x d y d z \\
& \int_{T} \int_{\partial S}\left(B_{x} d x d t+B_{y} d y d t+B_{z} d z d t\right) \\
&-\iint_{S}\left(E_{x} d y d z+E_{y} d z d x+E_{z} d x d y\right) \\
&=\iint_{T} \int_{S}\left(j_{x} d y d z d t+j_{y} d z d x d t+j_{z} d x d y d t\right) \\
& \int_{\partial V}\left(B_{x} d y d z+B_{y} d z d x+B_{z} d x d y\right)=0 \\
& \int_{T} \int_{\partial S}\left(E_{x} d x d t+E_{y} d y d t+E_{z} d z d t\right) \\
&+\int_{S}\left(B_{x} d y d z+B_{y} d z d x+B_{z} d x d y\right)=0 .
\end{aligned}
$$

Since the integration regions of these expressions are quite different, they do not show a clear way to grouping them, which is an important difference between the differential and integral formulations. For example, in Eq. (50), the elements of integration $d y d z, d z d x, d x d y$ etc. refer to a closed surface while those in Eq. (51) refer to an open surface. Furthermore, as time appears as a new variable of integration, the regions of integrations in space-time are more varied that in the three-dimensional space. The key point is to note that these elements can be chosen so that they were subsets of a same three-dimensional hyper-surface embedded in the four-dimensional space. We proceed as follows.

We first consider the inhomogeneous Maxwell equation (50). The LHS can be written as

$$
\begin{gathered}
\iint_{\partial V}\left(E_{x} d y d z+E_{y} d z d x+E_{z} d x d y\right)=\iint_{\partial V_{y z}} E_{x} d y d z \\
+\iiint_{\partial V_{z x}} E_{y} d z d x+\iiint_{\partial V_{x y}} E_{z} d x d y .
\end{gathered}
$$

Let us now consider the hyper-surface $\mathbf{H}_{S}=\mathbf{H}_{S}^{(1)} \cup \mathbf{H}_{S}^{(2)}$ where

$$
\begin{aligned}
& \mathbf{H}_{S}^{(1)}=\{(0, x, y, z) \mid(x, y, z) \in V\} \subset \mathbf{R}^{4}, \\
& \mathbf{H}_{S}^{(2)}=\{(t, x, y, z) \mid 0 \leq t \leq T ;(x, y, z) \in \partial V\} \subset \mathbf{R}^{4} .
\end{aligned}
$$

This hyper-surface is discussed in detail in Appendix B. $\mathbf{H}_{S}^{(2)}$ is an hyper-cylinder and $\mathbf{H}_{S}^{(1)}$ is one of its caps (the one 
at $t=0$ ). Thus, $\mathbf{H}_{S}$ is an open hyper-surface whose boundary $\partial \mathbf{H}_{S}$ is at $t=T$. We must observe that, unlike what was had in Subsecs. 2.2 and 2.3, now $\mathbf{H}_{S}$ is not totally arbitrary. The shape of this hyper-surface is dictated by the type of regions that are considered in the usual three-dimensional integral Maxwell equations. In abuse of the notation, the set $\mathbf{H}_{S}^{(1)}$ will be denoted as $(V), \partial \mathbf{H}_{S}^{(1)}$ as $(\partial V)$, and $\mathbf{H}_{S}^{(2)}$ as $(T \times \partial V)$. Hence, $\mathbf{H}_{S}=(V) \cup(T \times \partial V)$. Let $\mathbf{A}$ be an arbitrary set of $\mathbf{R}^{4}$, we will use the symbol $(\mathbf{A})_{x y z}$ to denote the region in the space $X Y Z$ that generates the projection of $\mathbf{A}$ on this space. The symbol $(\mathbf{A})_{x y}$ has a similar meaning. So, since the set $V$ generates the projection of $\mathbf{H}_{S}^{(1)}$ on the space $X Y Z$ we have: $\left(\mathbf{H}_{S}^{(1)}\right)_{x y z}=V,\left(\partial \mathbf{H}_{S}^{(1)}\right)_{x y z}=\partial V$, and $\left(\partial \mathbf{H}_{S}^{(1)}\right)_{x y}=\partial V_{x y}$. Then Eq. (54) can be written as

$$
\begin{gathered}
\iint_{\partial V}\left(E_{x} d y d z+E_{y} d z d x+E_{z} d x d y\right)=\iint_{\left(\partial \mathbf{H}_{S}^{(1)}\right)_{y z}} E_{x} d y d z \\
+\iint_{\left(\partial \mathbf{H}_{S}^{(1)}\right)_{z x}} E_{y} d z d x+\iint_{\left(\partial \mathbf{H}_{S}^{(1)}\right)_{x y}} E_{z} d x d y .
\end{gathered}
$$

It is clear than the more appropriate parameters to be used in the first integral of the RHS of this equation are $y$ and $z$. With this parametrization, the form of $d \sigma^{23}$ given by Eq. (22) is precisely $d y d z$ and, from Eq. (23), we have $d \phi_{01}=-d y d z$. Therefore, this integral can be written as

$$
\underset{\left(\partial \mathbf{H}_{S}^{(1)}\right)_{y z}}{\int} E_{x} d y d z=-\underset{\left(\partial \mathbf{H}_{S}^{(1)}\right)_{y z}}{ } E_{x} d \sigma_{01}=\iint_{\partial \mathbf{H}_{S}^{(1)}} F^{01} d \theta_{01}
$$

where we have used Eq. (3). The other integrals can be handled in a similar way. For the construction of Eq. (60) below, it is convenient to use the following antisymmetric matrix which shows the form of the elements of the tensor $d \theta_{\mu \nu}$ when they are expressed in the different parameterizations

$$
\left(\begin{array}{cccc}
0 & -d y d z & -d x d z & -d x d y \\
\cdots & 0 & d t d z & -d t d y \\
\cdots & \cdots & 0 & d t d x \\
\cdots & \cdots & \cdots & 0
\end{array}\right)
$$

One must to be aware, however, that this matrix is a mixture of the forms that the elements of the tensor $d \theta_{\mu \nu}$ have in the different parameterizations. Therefore, this matrix is not necessarily a tensor, a fact that can be easily proved. With these data the LHS of Eq. (50) becomes

$$
\begin{gathered}
\iint_{\partial V}\left(E_{x} d y d z+E_{y} d z d x+E_{z} d x d y\right) \\
=\iint_{\partial \mathbf{H}_{S}^{(1)}} F^{0 m} d \nabla_{0 m} .
\end{gathered}
$$

On the other hand, in the RHS of Eq. (50) there appear $d x d y d z$ which is the form of $d V^{123}$ when the parameters are $\left(u^{1}, u^{2}, u^{3}\right)=(x, y, z)$ Consequently, using Eq. (19), the RHS of Eq. (50) is equal to

$$
\begin{aligned}
\iint_{V} \int_{j^{0}} d x d y d z & =\iint_{\left(\mathbf{H}_{S}^{(1)}\right)_{x y z}} j^{0} d x d y d z \\
& =\iiint_{\mathbf{H}_{S}^{(1)}} j^{0} d x d y d z=\iiint_{\mathbf{H}_{S}^{(1)}} j^{0} d \mathbb{t} .
\end{aligned}
$$

Therefore, Eq. (50) can be written as

$$
\iint_{\partial \mathbf{H}_{S}^{(1)}} F^{0 m} d \sigma_{0 m}=\iiint_{\partial \mathbf{H}_{S}^{(1)}} F^{m 0} d \sigma_{m 0}=\iiint_{\mathbf{H}_{S}^{(1)}} j^{0} d \nabla_{0} .
$$

Note the equivalence between the integration regions. The last step is the key of the method followed in this subsection; it implies that the integration region $\partial V \subset \mathbf{R}^{3}$ generates the projection of $\partial \mathbf{H}_{S}^{(1)} \subset \mathbf{R}^{4}$. As in $\partial \mathbf{H}_{S}^{(1)}$ we have $d t=0$, it follows that $d \mathbb{\Psi}_{1}=d \mathbb{\downarrow}_{2}=d \mathbb{t}_{3}=0$ and the RHS of Eq. (62) can be written as $\iiint_{\partial \mathbf{H}_{S}^{(1)}} j^{\alpha} d \nabla_{\alpha}$. For the same reason (that is, $d t=0$ ) we have $d b_{12}=d \theta_{13}=d b_{23}=0$ and the LHS of Eq. (62) can be written as $\sum_{\mu} \sum_{\nu>\mu} F^{\mu \nu} d \theta_{\mu \nu}$. Therefore, the first inhomogeneous Maxwell equation (50) becomes

$$
\frac{1}{2} \iint_{\partial \mathbf{H}_{S}^{(1)}} F^{\alpha \beta} d \nabla_{\alpha \beta}=\iiint_{\mathbf{H}_{S}^{(1)}} j^{\alpha} d \nabla_{\alpha}
$$

We now consider the second inhomogeneous Maxwell equation (51). The open surface $S \subset \mathbf{R}^{3}$ appearing there will be chosen as follows: the closed surface $\partial V$ will be divided into two disjoint parts (except at the points of its boundaries), denoted as $S^{(T)}$ and $S^{(B)}$ So that $\partial V=S^{(T)} \cup S^{(B)}$ (for example, $S^{(T)}$ can be taken as the top part of $\partial V$ with respect to the $Z$ axis and $S^{(B)}$ as the bottom part). These two surfaces are open surfaces and expression (51) can be applied over any of them. Let us take for example $S=S^{(T)}$. Then, the LHS of Eq. (51) can be written as

$$
\begin{aligned}
\int_{T} \int_{\left(\partial S^{(T)}\right)_{x}} B_{x} d x d t & +\int_{T} \int_{\left(\partial S^{(T)}\right)_{y}} B_{y} d y d t+\iint_{T} \int_{\left(\partial S^{(T)}\right)_{z}} B_{z} d z d t \\
& -\iint_{\left(S^{(T)}\right)_{y z}} E_{x} d y d z+\iint_{\left(S^{(T)}\right)_{z x}} E_{y} d z d x \\
& -\iint_{\left(S^{(T)}\right)_{x y}} E_{z} d x d y .
\end{aligned}
$$

Let us consider the hyper-surfaces

$$
\begin{aligned}
& \mathbf{H}_{S}^{(T)}=\left\{(t, x, y, z) \mid 0 \leq t \leq T ;(x, y, z) \in S^{(T)}\right\} \subset \mathbf{R}^{4}, \\
& \mathbf{H}_{S}^{(B)}=\left\{(t, x, y, z) \mid 0 \leq t \leq T ;(x, y, z) \in S^{(B)}\right\} \subset \mathbf{R}^{4} .
\end{aligned}
$$


Comparing with the expression for $\mathbf{H}_{S}^{(2)}$ given in Eq. (55) we see that $\mathbf{H}_{S}^{(2)}=\mathbf{H}_{S}^{(T)} \cup \mathbf{H}_{S}^{(B)}$ and that the border of $\mathbf{H}_{S}^{(2)}$ matches the boundaries of $\mathbf{H}_{S}^{(T)}$ and $\mathbf{H}_{S}^{(B)}$ after deleting the common points. In addition, $S^{(T)}=\left(\partial \mathbf{H}_{S}^{(T)}\right)_{x y z}$ and $T \times\left(\partial S^{(T)}\right)_{x}=\left(\partial \mathbf{H}_{S}^{(T)}\right)_{x t}$. Therefore, for the first integral of expression (64) we have

$$
\begin{gathered}
\int_{T} \int_{\left(\partial S^{(T)}\right)_{x}} B_{x} d x d t=-\iint_{\left(\partial \mathbf{H}_{S}^{(T)}\right)_{t x}} F^{23} d x d t= \\
-\iint_{\partial \mathbf{H}_{S}^{(T)}} F^{23} d x d t=-\iint_{\partial \mathbf{H}_{S}^{(T)}} F^{23} d \theta_{23},
\end{gathered}
$$

where we have used the fact that $d \sigma^{10}=d x d t$ when the parameters are $\left(\theta^{1}, \theta^{2}\right)=(x, t)$. This implies $d \theta_{23}=-d \sigma^{01}=$ $d x d t$. For the second and third terms we have something similar. For the fourth term we have

$$
\begin{aligned}
& -\iint_{\left(S^{(T)}\right)_{y z}} E_{x} d y d z=-\iint_{\left(\partial \mathbf{H}_{S}^{(T)}\right)_{y z}} F^{10} d y d z \\
& =-\iint_{\partial \mathbf{H}_{S}^{(T)}} F^{10} d y d z=-\iint_{\partial \mathbf{H}_{S}^{(T)}} F^{01} d \wp_{01},
\end{aligned}
$$

and something similar for the fifth and sixth terms. Then the sum of the six terms of the LHS of Eq. (51) is equal to

$$
-\frac{1}{2} \iint_{\partial \mathbf{H}_{S}^{(T)}} F^{\alpha \beta} d \emptyset_{\alpha \beta} .
$$

Note again the equivalence between the integration regions. This implies that the integration region in space-time, denoted as $(T \times \partial S)$ is part of $\partial \mathbf{H}_{S}^{(T)}$.

On the other hand, we see that in the first integral on the RHS of Eq. (51) appears the element $d y d z d t$ which is the form of $d V^{230}$ when the parameters are $\left(u^{1}, u^{2}, u^{3}\right)=$ $(y, z, t)$. Consequently, using Eq. (19), that integral is equal to

$$
\begin{aligned}
\int_{T} \int_{S} j^{1} d y d z d t & =\iiint_{\left(\mathbf{H}_{S}^{(T)}\right)_{t y z}} j^{1} d y d z d t=\iiint_{\mathbf{H}_{S}^{(T)}} j^{1} d y d z d t \\
& =-\iiint_{\mathbf{H}_{S}^{(T)}} j^{1} d \psi_{1} .
\end{aligned}
$$

The other two integrals can be worked in the same way. Therefore the RHS of Eq. (51) is equal to

$$
-\iiint_{\mathbf{H}_{S}^{(T)}} j^{m} d \vec{W}_{m} .
$$

With these results, Eq.(51) is written as

$$
\frac{1}{2} \iint_{\partial \mathbf{H}_{S}^{(T)}} F^{\alpha \beta} d \Phi_{\alpha \beta}=\iiint_{\mathbf{H}_{S}^{(T)}} j^{m} d \mathbb{F}_{m} .
$$

It must be remarked one more time that Eqs. (19) and (23) are much more than just a way of writing the integration elements $d x^{m} d x^{n}$ and $d x^{l} d x^{o}$, they show a deeper fact: the integration regions $(S)$ associated with $d \sigma_{l o}$, and the region $(T \times \partial S)$, associated with $d \sigma_{m n}$, are parts of the same two- dimensional surface in the four-dimensional hyper-space; analogously, the regions $(V)$ and $(T \times \partial V)$ are parts of the same three-dimensional hyper-surface in the fourdimensional hyper-space. These facts are not evident from the integral Maxwell equations, written in their usual form.

It is clear that for the bottom hyper-surface $\partial \mathbf{H}_{S}^{(B)}$ we have the analogous result:

$$
\frac{1}{2} \iint_{\partial \mathbf{H}_{S}^{(B)}} F^{\alpha \beta} d \theta_{\alpha \beta}=\iiint_{\mathbf{H}_{S}^{(B)}} j^{m} d \nabla_{m} .
$$

Due to $\mathbf{H}_{S}^{(2)}=\mathbf{H}_{S}^{(T)} \cup \mathbf{H}_{S}^{(B)}$, the right members of Eqs. (72) and (73) can be grouped into a single integral over $\mathbf{H}_{S}^{(2)}$. Additionally, as the boundary of $\mathbf{H}_{S}^{(2)}$ matches the union of the boundary of $\mathbf{H}_{S}^{(T)}$ and $\mathbf{H}_{S}^{(B)}$ (after the common points have been deleted) and due to the contribution of those points in the integral over $\partial \mathbf{H}_{S}^{(T)} \cup \partial \mathbf{H}_{S}^{(B)}$ cancel themselves, we also have that left members of the two previous equations can be grouped into a single integral over $\partial \mathbf{H}_{S}^{(2)}$. Therefore, we have

$$
\begin{aligned}
& \frac{1}{2} \iint_{\partial \mathbf{H}_{S}^{(2)}} F^{\alpha \beta}=d \nabla_{\alpha \beta} \iiint_{\mathbf{H}_{S}^{(2)}} j^{m} d \Psi_{m} \\
& \text { where } \quad m=1,2,3 \quad \text { and } \quad \alpha, \beta=0,1,2,3
\end{aligned}
$$

Now, as in $\mathbf{H}_{S}^{(2)}$ we have $(x, y, z) \in \partial V$ it follows that $d V=0$ and $d \mathbb{V}_{0}=0$. As a consequence the integral $\iiint_{\mathbf{H}_{S}^{(2)}} j^{0} d \mathbb{W}_{0}$ is equal to zero and it can be added to the previous equation. So, the second inhomogeneous Maxwell equation (51) becomes

$$
\frac{1}{2} \iiint_{\partial \mathbf{H}_{S}^{(2)}} F^{\alpha \beta} d \nabla_{\alpha \beta}=\iiint_{\mathbf{H}_{S}^{(2)}} j^{\alpha} d \nabla_{\alpha} .
$$

Finally, since $\mathbf{H}_{S}=\mathbf{H}_{S}^{(1)} \cup \mathbf{H}_{S}^{(2)}$ Eqs. (63) and (74) can be grouped into a single equation. It suffices to note that the boundary of $\mathbf{H}_{S}$ matches the junctions of the boundaries of $\mathbf{H}_{S}^{(1)}$ y $\mathbf{H}_{S}^{(2)}$ (after the common points have been deleted) and that the contributions of those points in the integral over $\partial \mathbf{H}_{S}^{(1)} \cup \partial \mathbf{H}_{S}^{(2)}$ cancel themselves. Therefore,

$$
\frac{1}{2} \iint_{\partial \mathbf{H}_{S}} F^{\alpha \beta} d \nabla_{\alpha \beta}=\iiint_{\mathbf{H}_{S}} j^{\alpha} d \boldsymbol{\psi}_{\alpha},
$$

This equation is Eq. (26), obtained in Subsec. 2.2 except that now $\mathbf{H}_{S}$ is not totally arbitrary. This is so because 
to derive Eq. (75) we have used regions whose geometric characteristics are appropriate for the experimental observation of electromagnetic phenomena. On the other hand, to derive Eq. (26), $\mathbf{H}_{S}$ had no restrictions. Nevertheless, one of the most important implications of having demonstrated Eq. (75) is that the usual integral expressions are consistent with the general covariant integral formulation of Eq. (26).

It can be shown that for the type of hyper-surfaces such as those considered in Eq. (75) the integral of the RHS is equal to zero even though $j^{\alpha}$ is different from zero (see Appendix B). Therefore, it is necessary to look for another approach as will be done in the next section where we use a different method.

The homogenous Maxwell equations, (52) and (53) can be expressed in an explicitly covariant form if we take into account that, for $\rho=0$ and $\mathbf{j}=0$ Eqs. (50) and (51) are identical to Eqs. (52) and (53) after the transformations (EM duality)

$$
\begin{aligned}
& \mathbf{E} \rightarrow \mathbf{B}, \\
& \mathbf{B} \rightarrow-\mathbf{E} .
\end{aligned}
$$

This transformation is equivalent to the one that transforms the tensor $F^{\mu \nu}$ into its dual tensor $\mathfrak{F}^{\mu \nu}$ defined in Eq. (16). Therefore, the expression

$$
\iint_{\Omega(2)} \mathfrak{F}^{\mu \nu} d \theta_{\mu \nu}=0
$$

represents the homogeneous ECIME.

We can see now that the procedure to show the covariance of the integral Maxwell equations is much more complicated than the one to show the covariance of the differential equations.

To end this section, it is convenient to make the following comments which, although trivial, complement the discussion.

- In the previous developments we have started from a certain form of the Maxwell's equations and have arrived at their covariant integral form. But one can also do the inverse, i.e., to derive the other forms of the Maxwell equations starting from the covariant integral form.

- Since the covariant integral equations are expressed in terms of tensors with respect to the Lorentz transformation, their form will be the same in any inertial reference frame. Therefore, since the set of covariant integral equations implies the other forms, it is concluded that the integrals over the transformed regions will give the transformed Maxwell equations in the new reference system.

- Two different inertial observers will assign different values to the functions $F^{\mu \nu}$ and $j_{\mu}$. But regardless of the system of reference inertial used, the behavior of these functions will be governed by the same equations.

\section{Maxwell equations integrated over less usual regions}

\subsection{Non covariant case}

The region of integration used in the previous section, as mentioned before, are not the only ones. Therefore, the integral Maxwell equations (44)-(47) are not the only ones. As we will see below there is a more convenient way to express them to make the transition to an explicitly covariant formulation easier. To see this point we remember the usual way of obtaining the Maxwell integral equations from the differential ones (8), (9), (11) and (12).

To transform (8) and (11) into (44) and (46), a volume integral is made, after which the divergence theorem is applied. On the other hand, to pass from (9) and (12) to (45) and (47), a surface integral is made and then Stokes theorem is applied. The time derivatives are not touched at all. In other words, there is no time integration. Therefore, it is clear that this procedure is far from being symmetric in space and time, as relativity theory demands. Thus, it is necessary to proceed in another way, trying to put the time and space coordinates on the same level. We proceed as follows.

We first make a volume integration of all the Eqs. (8), (9), (11) and (12). In the equations that involve divergences we use the divergence theorem to pass from a volume integral to a surface integral, as usual. In equations involving the rotational we use the following corollary of the Stokes theorem

$$
\iiint_{V} \nabla \times \mathbf{G} d V=\iint_{\partial V} d \mathbf{S} \times \mathbf{G}
$$

Thus, Eqs. (9) and (12) transform into

$$
\iint_{\partial V} d \mathbf{S} \times \mathbf{B}-\frac{d}{d t} \iiint_{V} \mathbf{E} d V=\iiint_{V} \mathbf{J} d V
$$

and

$$
\iint_{\partial V} d \mathbf{S} \times \mathbf{E}+\frac{d}{d t} \iiint_{V} \mathbf{B} d V=0
$$

It is now clear that it is necessary to eliminate the time derivatives by integration. The results are

$$
\int_{T} d t \iint_{\partial V} d \mathbf{S} \times \mathbf{B}-\iiint_{V} \mathbf{E} d V=\int d t \iiint_{V} \mathbf{j} d V,
$$

and

$$
\int_{T} d t \iint_{\partial V} d \mathbf{S} \times \mathbf{E}+\iiint_{V} \mathbf{B} d V=0
$$

This pair of equations, together with the pair (44), (46) is an equivalent form of writing the integral Maxwell equations. 
As we will see below, they have significant advantages that let us to pass to the covariant formulation. This also shows another perspective of electromagnetic theory. Because our aim is to achieve complete symmetry in space-time, before writing Eqs. (44), (46), (81) and (82) in terms of Cartesian coordinates, it is worthwhile to remark that the LHS term of (44) and (46) involve surface integrations. Thus, we have to deal with two surfaces and three-dimensional hyper-surfaces. In order to have more symmetry in Eqs. (44) and (46), we integrate over time, obtaining

$$
\iint_{T} \int_{\partial V} \mathbf{E} \square d \mathbf{S} d t=\iiint_{T} \int_{V} \rho d V d t
$$

and

$$
\int_{T} \iint_{\partial V} \mathbf{B} \square d \mathbf{S} d t=0 .
$$

Then, the Maxwell equations in other of their integral forms are

$$
\begin{aligned}
\int_{T} \int_{\partial V} \int_{V} \mathbf{E} \square d \mathbf{S} d t & =\iiint_{T} \rho d V d t, \\
\int_{T} d t \iint_{\partial V} d \mathbf{S} \times \mathbf{B}-\iiint_{V} \mathbf{E} d V & =\int d t \iiint_{T} \mathbf{j} d V, \\
\int_{T} \iint_{\partial V} \mathbf{B} \square d \mathbf{S} d t & =0,
\end{aligned}
$$

and

$$
\int_{T} d t \iint_{\partial V} d \mathbf{S} \times \mathbf{E}+\iiint_{V} \mathbf{B} d V=0 .
$$

Thus, having different forms of writing the integral Maxwell equations raises the question of which of those forms can suggest a better way to show the explicit covariance.

\subsection{Covariant integral case starting from the three- dimensional differential expressions}

We begin by writing the non-covariant integral Maxwell equations (85)-(86) in Cartesian coordinates,

$$
\begin{gathered}
\int_{T} \int_{\partial V}\left(E_{x} d y d z+E_{y} d z d x+E_{z} d x d y\right) d t \\
=\iiint_{T} \rho d x d y d z d t, \\
\int_{T} \int_{\partial V}\left(B_{z} d z d x-B_{y} d y d x\right) d t-\iiint_{V} E_{x} d x d y d z \\
=\iiint_{T} \int_{V} j_{x} d x d y d z d t, \\
\int_{T} \int_{\partial V}\left(B_{x} d y d x-B_{z} d z d y\right) d t-\iint_{V} E_{y} d x d y d z \\
=\iiint_{T} j_{y} d x d y d z d t, \\
\underset{T}{\int} \int_{\partial V}\left(B_{y} d y d z-B_{x} d x d z\right) d t-\iint_{V} E_{z} d x d y d z \\
=\iint_{T} \iint_{V} j_{z} d x d y d z d t .
\end{gathered}
$$

It is evident from these equations that integration regions in the LHS of all these equations are of the same type, which makes easier to the passage to the covariant formulation. Now, taking the space-time volume as usual,

$$
d^{4} x=d x d y d z d t .
$$

The RHS of Eqs. (89) and (90) can be written as

$$
\left.\begin{array}{l}
\iiint \int_{T \times V} \rho d^{4} x \\
\iiint_{T \times V} j_{x} d^{4} x \\
\iiint_{T \times V} j_{y} d^{4} x \\
\iiint_{T \times V} j_{z} d^{4} x
\end{array}\right\} \rightarrow \iiint \int_{V} j^{\mu} d^{4} x
$$

where we have introduced a new symbol $\mathbf{V}$ to denote a hyper-volume. As was the case in the last section again, the key point is to recognize that both, $(T \times V)$ and $(V)$, are parts of $\mathbf{V}$.

Using Eqs. (7) and (19) we can express the LHS of (89) in the form

$$
\begin{aligned}
\int_{T} \int_{\partial V} \int_{\partial} & E_{x} d y d z+E_{y} d z d x \\
+ & \left.E_{z} d x d y\right) d t \rightarrow \iiint_{\partial V} F^{0 n} d V_{n}
\end{aligned}
$$


while for the LHS of Eq. (90), using Eq. (19), we obtain

$$
\begin{aligned}
\iint_{T} \iint_{\partial V}( & \left.B_{z} d z d x-B_{y} d y d x\right) d t-\iiint_{V} E_{x} d x d y d z \\
& =\iiint_{T \times \partial V} \varepsilon^{l m n} d V_{m} B_{n}+\iiint_{V} F^{10} d V_{0} \\
& =\iiint_{T \times \partial V} \varepsilon^{l m n} d V_{m}\left(-\frac{1}{2} \varepsilon_{n i j} F^{i j}\right)+\iiint_{V} F^{10} d V_{0} \\
& =\iiint_{T \times \partial V} F^{l m} d V_{m}+\iiint_{V} F^{10} d V_{0} .
\end{aligned}
$$

The left-hand side of Eqs. (91) and (92) can be written similarly. Thus, the left hand side of Eqs. (90)-(92) can be written generically as

$$
\iiint_{T \times \partial V} F^{l m} d V_{m}+\iiint_{V} F^{10} d V_{0},
$$

which clearly is the component $\mu=m$ of

$$
\iiint_{(V)=\partial V} F^{\mu \nu} d V_{\nu}
$$

while Eq. (95) is the $\mu=0$ component. Then, expression (98), with the result given in Eq. (94), permits us to write the ECIME in the form

$$
\iiint_{(V)=\partial \boldsymbol{V}} F^{\mu \nu} d V_{\nu}=\iiint \int_{\boldsymbol{V}} j^{\mu} d^{4} x,
$$

According to usual conventions, we can write the threevolume of space-time as the hyper-surface element $d \sigma_{\nu}$

$$
d V_{\nu}=d \sigma_{\nu}
$$

Then we have a hyper-surface integral of a second-rank space-time tensor related to the hyper-volume integral of the four-vector density current, that is,

$$
\iiint_{(V)=\partial \boldsymbol{V}} F^{\mu \nu} d \sigma_{\nu}=\iiint \int_{\boldsymbol{V}} j^{\mu} d^{4} x .
$$

The homogeneous ECIME can be obtained by means of the dual field tensor $\mathfrak{F}^{\mu \nu}$. So Eqs. (87) and (88) can be written as

$$
\iiint_{\partial V} \mathfrak{F}^{\mu \nu} d \sigma_{\nu}=0
$$

\subsection{Covariant integral case starting from the four- dimensional differential expressions}

We first multiply Eq. (10) by the hyper volume element $d^{4} x$ and then integrate to obtain

$$
\iiint \int_{\boldsymbol{V}} \partial_{\mu} F^{\mu \nu} d^{4} x=\iiint \int_{\boldsymbol{V}} j^{\nu} d^{4} x .
$$

We now transform the LHS of Eq. (91). Using the Stokes theorem, one obtains

$$
\iiint \int_{\boldsymbol{V}} \partial_{\mu} F^{\mu \nu} d^{4} x=\iiint \int_{\boldsymbol{V}} j^{\nu} d^{4} x .
$$

Which corresponds to Eq. (87). The homogeneous Eqs. (90) can be obtained in a similar way.

As we already mentioned, the form of writing the covariant Maxwell equations in their integral form is not unique; this is clearer when we depart from the explicitly covariant formulation of the Maxwell differential equations.

\section{Concluding remarks}

We have discussed several ways to obtain ECIME. In the case in which we started from the IME3 it was necessary to express the usual integral expressions without time derivatives to obtain a true set of integral equations. Our analysis also shows the difficulties that one faces in the process of choosing the hyper-surfaces on which it is necessary to integrate. This way is not as straightforward as the one in which one directly integrates the explicitly covariant form of the differential equations. It must be remarked that we proposed an alternative form of writing the integral Maxwell equations, both non-covariantly and explicitly covariantly. Our treatment also opens the perspective of seeing the integral equations of Maxwell in the structure of space-time, that is objective of relativity theory.

\section{Appendix A.}

Definition. Let us consider a hyper-surface $\mathbf{H}_{S} \subset \mathbf{R}^{4}$ generated by the parametric four-vector equation (for simplicity we assume that $\mathbf{H}_{S}$ is generated by only one equation of this type, but the generalization for when $\mathbf{H}_{S}$ is generated by two o more equations of this type is obvious)

$$
\overrightarrow{\mathbf{x}}=\overrightarrow{\mathbf{X}}(\mathbf{u})
$$

where $\overrightarrow{\mathbf{x}}=\left(x^{\mu}\right)=\left(x^{0}, x^{1} x^{2}, x^{3}\right)$ is the four-vector defined in Eq. (1), $\overrightarrow{\mathbf{X}}(\mathbf{u})=\left(X^{0}(\mathbf{u}), X^{1}(\mathbf{u}), X^{2}(\mathbf{u}), X^{3}(\mathbf{u})\right)$ is a fourcomponent function depending on the three-dimensional variable $\mathbf{u}=(u, v, w) \in V_{\mathbf{u}}$ being $V_{\mathbf{u}} \subset \mathbf{R}^{3}$ the threedimensional region where the parameters $u, v, w$ take values. Let us consider a function $\phi$ defined in $\mathbf{H}_{S}$.

The hyper-surface integral $\iiint_{\mathbf{H}_{S}} \phi d \mathbb{W}_{\delta}$ of the function $\phi$ over the hyper-surface $\mathbf{H}_{S}$ is defined as

$$
\begin{aligned}
\iiint_{\mathbf{H}_{S}} \phi d \vec{\forall}_{\delta} & \equiv \iiint_{V_{\mathbf{u}}} \phi(\overrightarrow{\mathbf{X}}(\mathbf{u})) \\
& \times\left[\varepsilon_{\alpha \beta \gamma \delta} \frac{\partial X^{\alpha}}{\partial u} \frac{\partial X^{\beta}}{\partial u} \frac{\partial X^{\gamma}}{\partial w} d V_{\mathbf{u}}\right]
\end{aligned}
$$


where the symbols $\phi(\overrightarrow{\mathbf{X}}(\mathbf{u}))$ and

$$
\varepsilon_{\alpha \beta \gamma \delta} \frac{\partial X^{\alpha}}{\partial u} \frac{\partial X^{\beta}}{\partial u} \frac{\partial X^{\gamma}}{\partial w} d V_{\mathbf{u}}
$$

are the values of $\phi$ and $d \downarrow$ on the hyper-surface $\mathbf{H}_{S}$ respectively. Explicitly

$$
\begin{aligned}
& \iiint_{\mathbf{H}_{S}} \phi d \vec{\forall}_{0} \equiv \iiint_{V_{\mathbf{u}}} \phi(\overrightarrow{\mathbf{X}}(\mathbf{u})) \\
& \times\left|\begin{array}{ccc}
\frac{\partial X^{1}}{\partial u} & \frac{\partial X^{2}}{\partial u} & \frac{\partial X^{3}}{\partial u} \\
\frac{\partial X^{1}}{\partial v} & \frac{\partial X^{2}}{\partial v} & \frac{\partial X^{3}}{\partial v} \\
\frac{\partial X^{1}}{\partial w} & \frac{\partial X^{2}}{\partial w} & \frac{\partial X^{3}}{\partial w}
\end{array}\right| \\
& \iiint_{\mathbf{H}_{S}} \phi d W_{1} \equiv-\iiint_{V_{\mathbf{u}}} \phi(\overrightarrow{\mathbf{X}}(\mathbf{u})) \\
& \times\left|\begin{array}{ccc}
\frac{\partial X^{2}}{\partial u} & \frac{\partial X^{3}}{\partial u} & \frac{\partial X^{0}}{\partial u} \\
\frac{\partial X^{2}}{\partial v} & \frac{\partial X^{3}}{\partial v} & \frac{\partial X^{0}}{\partial v} \\
\frac{\partial X^{2}}{\partial w} & \frac{\partial X^{3}}{\partial w} & \frac{\partial X^{0}}{\partial w}
\end{array}\right| d V_{\mathbf{u}} \\
& \iiint_{\mathbf{H}_{S}} \phi d \mathbb{V}_{2} \equiv \iiint_{V_{\mathbf{u}}} \phi(\overrightarrow{\mathbf{X}}(\mathbf{u})) \\
& \times\left|\begin{array}{ccc}
\frac{\partial X^{3}}{\partial u} & \frac{\partial X^{0}}{\partial u} & \frac{\partial X^{1}}{\partial u} \\
\frac{\partial X^{3}}{\partial v} & \frac{\partial X^{0}}{\partial v} & \frac{\partial X^{1}}{\partial v} \\
\frac{\partial X^{3}}{\partial w} & \frac{\partial X^{0}}{\partial w} & \frac{\partial X^{1}}{\partial w}
\end{array}\right| d V_{\mathbf{u}} \\
& \iiint_{\mathbf{H}_{S}} \phi d \nabla_{3} \equiv-\iiint_{V_{\mathbf{u}}} \phi(\overrightarrow{\mathbf{X}}(\mathbf{u})) \\
& \times\left|\begin{array}{ccc}
\frac{\partial X^{0}}{\partial u} & \frac{\partial X^{1}}{\partial u} & \frac{\partial X^{2}}{\partial u} \\
\frac{\partial X^{0}}{\partial v} & \frac{\partial X^{1}}{\partial v} & \frac{\partial X^{2}}{\partial v} \\
\frac{\partial X^{0}}{\partial w} & \frac{\partial X^{1}}{\partial w} & \frac{\partial X^{2}}{\partial w}
\end{array}\right| d V_{\mathbf{u}}
\end{aligned}
$$

For example, for the special case in which the parameters are $x=u, y=v, z=w$ Eq. (A3) becomes

$$
\iiint_{\mathbf{H}_{S}} \phi d \mathbb{F}_{0} \equiv \iiint_{V} \phi\left(X^{0}(x, y, z), x, y, z\right) d x d y d z .
$$

Appendix B.

In this appendix we demonstrate that for certain hypersurfaces the RHS of Eq. (20) can be equal to zero even though the sources are different from zero.

Let us consider the hyper-surface

$$
\mathbf{H}_{S}=\mathbf{H}_{S}^{(1)} \cup \mathbf{H}_{S}^{(2)},
$$

where

$$
\begin{aligned}
& \mathbf{H}_{S}^{(1)}=\{(0, x, y, z) \mid(x, y, z) \in V\} \subset \mathbf{R}^{4}, \\
& \mathbf{H}_{S}^{(2)}=\{(t, x, y, z) \mid 0 \leq t \leq T ;(x, y, z) \in \partial V\} \subset \mathbf{R}^{4},
\end{aligned}
$$

here $V \subset \mathbf{R}^{3}$ is a three-dimensional volume independent from $t$ and $\partial V$ is the surface surrounding $V$. In abuse of the notation the set $\mathbf{H}_{S}^{(1)}$ will be denoted as $(V) \subset \mathbf{R}^{4}$ and $\mathbf{H}_{S}^{(2)}$ as $(T \times \partial V) \subset \mathbf{R}^{4}$. So, $\mathbf{H}_{S}=(V) \cup(T \times \partial V)$.

The hyper-surface $\mathbf{H}_{S}^{(2)}$ is a hyper-cylinder and $\mathbf{H}_{S}^{(1)}$ is one of its caps (the one at $t=0$ ). For the case in which the hyper-surface $\mathbf{H}_{S}$ does not contain none of its caps the expression for $\mathbf{H}_{S}$ reduces to $\mathbf{H}_{S}^{(2)}$.

Let $\mathbf{C}$ an arbitrary set of $\mathbf{R}^{4}$ we will use the symbol $(\mathbf{C})_{x y z}$ to denote the region in the space $X Y Z$ that generates the projection of $\mathbf{C}$ on this space. The symbol $(\mathbf{C})_{x y}$ has an similar meaning.

So, since the set $V$ generates the projection of $\mathbf{H}_{S}$ on the space $X Y Z$ we have

$$
\begin{aligned}
\left(\mathbf{H}_{S}\right)_{x y z} & =V, \quad\left(\partial \mathbf{H}_{S}\right)_{x y z}=\partial V, \\
\text { and } \quad\left(\partial \mathbf{H}_{S}\right)_{x y} & =\partial V_{x y} .
\end{aligned}
$$

Assigning a similar meaning for the symbols $\left(\mathbf{H}_{S}\right)_{t x y}$, $\left(\mathbf{H}_{S}\right)_{t x z},\left(\mathbf{H}_{S}\right)_{t y z}$ we have

$$
\begin{aligned}
& \left(\mathbf{H}_{S}\right)_{t x y}=T \times \partial V_{x y}, \\
& \left(\mathbf{H}_{S}\right)_{t x z}=T \times \partial V_{x z}, \\
& \left(\mathbf{H}_{S}\right)_{t y z}=T \times \partial V_{y z},
\end{aligned}
$$

where $\partial V_{i j}$ is the projection of $\partial V$ on the plane $I J$. Then, the RHS of Eq. (20) becomes

$$
\begin{aligned}
\iiint_{\mathbf{H}_{S}} j^{\nu} d \mathbb{W}_{\nu} & =\iiint_{\mathbf{H}_{S}} \rho d x d y d z+\iiint_{\mathbf{H}_{S}} j_{x} d y d z d t \\
& +\iiint_{\mathbf{H}_{S}} j_{y} d x d z d t+\iiint_{\mathbf{H}_{S}} j_{z} d x d y d t .
\end{aligned}
$$

The first integral of this expression is equal to (notice that this term does not appear when $\mathbf{H}_{S}$ does not include its cap)

$$
\begin{gathered}
\iiint_{\mathbf{H}_{S}} \rho d x d y d z=\iiint_{\left(\mathbf{H}_{S}\right)_{x y z}} \rho d x d y d z=\iiint_{V} \rho d x d y d z=Q, \\
(Q=\text { charge inside } V),
\end{gathered}
$$


while for the second integral of the RHS of Eq. (B.8) we have

$$
\begin{aligned}
& \iiint_{\mathbf{H}_{S}} j_{x} d y d z d t=\iiint_{\left(\mathbf{H}_{S}\right)_{t y z}} j_{x} d y d z d t=\iiint_{T \times \partial V_{y z}} j_{x} d y d z d t \\
& =\int_{0}^{T}\left[\iint_{\partial V_{y z}} j_{x} d y d z\right] d t=\int_{0}^{T}\left[\iint_{\partial V} j_{x} d y d z\right] d t .
\end{aligned}
$$

We now use the formulation above Eq. (32). Thus,

$$
\begin{aligned}
\iiint_{\mathbf{H}_{S}} j_{x} d y d z d t & =\int_{0}^{T}\left[\iint_{\partial V} j_{x} \cos \alpha d S\right] d t \\
& =\int_{0}^{T}\left[\iint_{\partial V} j_{x} n_{1} d S\right] d t .
\end{aligned}
$$
So

The remaining terms of (B.8) give rise to a similar result.

$$
\begin{aligned}
\iint_{\mathbf{H}_{S}} j^{\nu} d \boldsymbol{T}_{\nu} & =Q+\int_{0}^{T}\left[\iint_{\partial V} \mathbf{j} \cdot \hat{\mathbf{n}} d S\right] d t \\
& =Q+\int_{0}^{T}\left[\iiint_{V} \nabla \cdot \mathbf{j} d V\right] d t \\
& =Q-\int_{0}^{T}\left[\iint_{V} \frac{\partial \rho}{\partial t} d V\right] d t \\
& =Q-\int_{0}^{T} \frac{d}{d t}\left[\iint_{V} \rho d V\right] d t=0,
\end{aligned}
$$

where we have used the fact that $V$ is independent from $t$ and $Q=Q(t)$. For the case when the hyper-surface does not include its cap, the first term of Eq. (B.12) not appear and the result is

$$
\iiint_{\mathbf{H}_{S}} j^{\nu} d \nabla_{\nu}^{\nu}=-Q
$$

For more details see Ref. [20] where D. van Dantzig analyzed this problem in 1934 from a different perspective. From the previous discussion is evident the essential role that an adequate handling of hyper-surfaces plays.

\section{Acknowledgments}

The authors express their gratitude to I. Campos and G Hernández for their careful reading of the manuscript and for valuable discussions.
1. M. Bunge, Foundations of Physics (Springer, Berlin, 1969). https://doi.org/10.1007/978-3-642-49287-7

2. V. D. Barger and M. G. Olsson, Classical Electricity and Magnetism (Allyn and Bacon, Newton, Massachusetts, 1987).

3. D. Griffiths, Introduction to Electrodynamics, 3rd. ed. (Prentice-Hall, New Jersey, 1999).

4. M. A. Heald and J.B. Marion, Classical Electromagnetic Radiation, 3rd ed. (Saunders College Publishing, Florida).

5. H. C. Ohanian, Classical Electrodynamics, (Allyn and Bacon, Massachusetts, 1988).

6. M. Schwartz, Principles of Electrodynamics (McGraw Hill, New York, 1972).

7. J. D. Jackson, Classical Electrodynamics, 3rd ed. (Wiley, New York, 1999).

8. J. Vanderline, Classical Electromagnetic Theory, (Springer, Dordrecht, 2004), https://doi.org/10.1007/ 1-4020-2700-1.
9. B. Di Bartolo, Classical Theory of Electromagnetism 2nd ed. (World Scientific, Singapore, 2004).

10. F. E. Low, Classical Field Theory. Electromagnetism and Gravitation, (Wiley, New York, 1997). https : //doi .org/10. $1002 / 9783527617456$.

11. D. F. Lawden, An Introduction to Tensor Calculus (Methuen, London, 1967).

12. W. Pauli, Theory of Relativity, (Dover, New York, 1981).

13. C. Møller, The Theory of Relativity (Oxford University Press, Oxford, 1974).

14. J. L. Anderson, Principles of Relativity Physics, (Academic Press, New York, 1967).

15. J. Aharoni, The Special Theory of Relativity, 2nd ed. (Oxford University Press, Oxford, 1965).

16. E. Ley Koo, Maxwell equations in Lorentz covariant integral form, Rev. Mex. Fis. E 52 (2006) 84.

17. A. Kovetz, The Principles of Electromagnetic Theory (Cambridge University Press, Cambridge, 1990). 
18. Perhaps the most familiar form of the divergence theorem is $\iiint_{V} \nabla \square \mathbf{F} d V=\emptyset \int_{\partial V} \mathbf{F} \square \mathbf{n} d \sigma$ where $V$ is an arbitrary volume, $\partial V$ the closed surface that surrounds it, and $\mathbf{F}$ a vector valued function. If, for example, this theorem is applied to the function $\mathbf{F}=(\phi, 0,0)$ one obtains $\iiint_{V} \partial \phi / \partial x d V=$ $\square \int_{\partial V} \phi d \sigma_{x y}$ where $d \sigma_{x y}=n_{1} d \sigma$ is the projection of $d \sigma$ on the plane $X Y$. When this surface integral is evaluated using the parameters $x, y$ the projection $d \sigma_{x y}$ is equal to $d x d y$ (see Ref. [19]).
19. G. Monsivais and S. de Neymet, Teoremas de Green, Gauss y Stokes para funciones continuas y discontinuas, $2 \mathrm{nd}$ ed. (La Prensa de Ciencias, México, 2013).

20. D. van Dantzig, The fundamental equations of electromagnetism, independent of metrical geometry, Math. Proc. Camb. Philos. Soc. 30 (1934) 421, https: / / doi .org/10. $1017 /$ S0305004100012664 